\title{
TARPSY: nouvelle fonction pour les rapports de sortie
}

\section{Jürg Unger-Köppel}

Dr méd., membre du Comité central de la FMH, responsable du département Médecine et tarifs hospitaliers

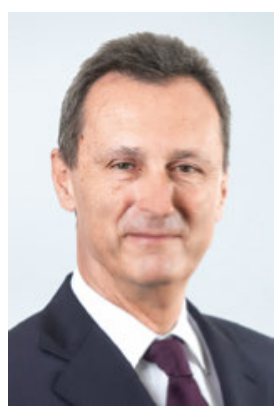

A partir du $1^{\text {er }}$ janvier 2018, la psychiatrie hospitalière de l'adulte sera facturée selon le nouveau système tarifaire TARPSY. La psychiatrie connaîtra donc aussi le passage des forfaits journaliers classiques à un nouveau système de rémunération journalière dégressif en fonction du diagnostic. En rapprochant le lien entre la prestation et son indemnisation, ce système répond à une exigence de la loi sur l'assurance-maladie (LAMal). Récemment, les partenaires tarifaires ont approuvé la convention de la nouvelle structure tarifaire, qui spécifie, entre autres, comment réagir si une institution a des revenus très supérieurs ou très inférieurs après le changement de système. C'est aussi la dernière condition qui devait être remplie pour que le Conseil fédéral puisse valider le tarif dans les temps pour une introduction au $1^{\text {er }}$ janvier 2018.

\section{Le diagnostic et les différents symptômes auront un impact direct sur la rémunération.}

Swiss Mental Health Care (SMHC), l'association regroupant les cliniques et hôpitaux psychiatriques de l'ensemble de la Suisse, a organisé fin juin 2017 un congrès consacré à la préparation des institutions à l'introduction du TARPSY. Ce congrès, qui a connu une forte participation, a montré que le corps médical et l'administration avaient encore passablement de questions à résoudre d'ici le début 2018. Les responsables des négociations avec les caisses-maladie ne peuvent pas s'attendre à ce que le forfait journalier d'aujourd'hui soit simplement converti en prix de base (base rate). Ils doivent présenter des calculs beaucoup plus complexes s'ils veulent obtenir de bons résultats pour leurs institutions lors des négociations. Mais le corps médical aussi devra bien se préparer: jusqu'à présent dans le quotidien des institutions, il est peu probable que des équipes autres que le médecincadre et le médecin-assistant aient étudié de près le diagnostic du patient tant qu'il y avait une amélioration clinique. Cela va désormais changer car le diagnostic et les différents symptômes auront un impact direct sur la rémunération. Les cliniques psychiatriques percevront en effet plus ou moins d'argent selon le diagnostic clinique posé. Cette relation directe avec l'indemnisation n'existait pas sous le régime des forfaits journaliers classiques. Le forfait était le même pour tous les diagnostics. Ce changement de système a déjà été appliqué aux soins somatiques aigus lors de l'introduction des DRG en 2012.

En raison de la pression qui règne un peu partout sur les recettes, les institutions pourraient être tentées de poser de préférence les diagnostics qui rapportent le plus. C'est pourquoi une révision du codage sera aussi mise en place. Lors de contrôles inopinés, une équipe d'externes indépendants vérifiera, sur la base des mêmes documents à la disposition des codeurs, si le codage est plausible. Un rapport sur la qualité du codage sera ensuite accessible à l'institution concernée ainsi qu'aux caisses et aux cantons. L'objectif déclaré des responsables de la psychiatrie est de faire en sorte que le codage et la révision du codage soient réalisés sur la seule base du rapport de sortie, et non sur la base de tout l'historique médical du patient avec des détails intimes, qui ne sont pas décisifs pour la pose du diagnostic. De ce fait, le rapport de sortie en psychiatrie accède à une deuxième fonction: en plus des informations essentielles pour le suivi du patient, il doit désormais contenir toutes les informations permettant de garantir un codage correct. Il est recommandé que le

\section{Le rapport de sortie ne réunit pas seulement} les informations nécessaires au suivi du traitement, il constitue aussi la base du codage.

corps médical, sous la direction des médecins-chefs, profite du temps qui reste jusqu'à la fin de l'année pour revoir en profondeur le processus rédactionnel des rapports de sortie et garantir que ces rapports répondent aux nouvelles exigences. La pratique décidera si les soignants en charge du suivi recevront un exemplaire complet ou seulement un extrait du rapport détaillé servant au codage et à sa révision. 